\title{
Evaluation of Lumbar Intervertebral Disc Degeneration Using T $1 \rho$ and T2 Magnetic Resonance Imaging in a Rabbit Disc Injury Model
}

\author{
Tetsuhiro Ishikawa ${ }^{1, *}$, Atsuya Watanabe ${ }^{2}$, Hiroto Kamoda ${ }^{1, *}$, \\ Masayuki Miyagi ${ }^{1,}{ }^{*}$, Gen Inoue ${ }^{1}$, Kazuhisa Takahashi ${ }^{1}$, Seiji Ohtori ${ }^{1}$ \\ ${ }^{1}$ Department of Orthopaedic Surgery, Graduate School of Medicine, Chiba University, Chiba, Japan \\ ${ }^{2}$ Department of Orthopaedic Surgery, Teikyo University Chiba Medical Center, Ichihara, Japan
}

\begin{abstract}
Study Design: An in vivo histologic and magnetic resonance imaging (MRI) study of lumbar intervertebral disc (IVD) degeneration was conducted.

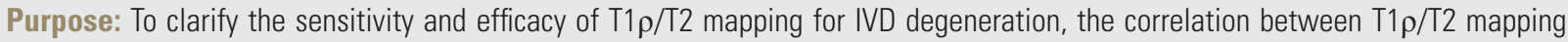
and degenerative grades and histological findings in the lumbar IVD were investigated.

Overview of Literature: The early signs of IVD degeneration are proteoglycan loss, dehydration, and collagen degradation. Recently, several quantitative MRI techniques have been developed; T2 mapping can be used to evaluate hydration and collagen fiber integrity within cartilaginous tissue, and T1 $\rho$ mapping can be used to evaluate hydration and proteoglycan content.

Methods: Using New Zealand White rabbits, annular punctures of the IVD were made 10 times at L2/3, 5 times at L3/4, and one time at $L 4 / 5$ using an 18-gauge needle $(n=6)$ or a 21-gauge needle $(n=6)$. At 4 and 8 weeks post-surgery, MRI was performed including T1 $\rho$ and T2 mapping. The degree of IVD degeneration was macroscopically assessed using the Thompson grading system. All specimens were cut for hematoxylin and eosin, safranin-0, and toluidine blue staining.

Results: Disc degeneration became more severe as the number of punctures increased and when the larger needle was used. $T 1 \rho$ and T2 values were significantly different between grade 1 and grade 3 IVDs, grade 1 and grade 4 IVDs, grade 2 and grade 3 IVDs, and grade 2 and grade 4 IVDs $(p<0.05)$. There was a significant difference between grade 1 and grade 2 IVDs only in terms of T1 $\rho$ values $(p<0.05)$.

Conclusions: $T 1 \rho$ and T2 quantitative MRI could detect these small differences. Our results suggest that $T 1 \rho$ and $T 2$ mapping are sensitive to degenerative changes of lumbar IVDs and that T1 $\rho$ mapping can be used as a clinical tool to identify early IVD degeneration.
\end{abstract}

Keywords: Intervertebral disc degeneration; Magnetic resonance imaging; Animal experiment; Histology

\section{Introduction}

Low back pain is a common and significant clinical and socioeconomic problem; it can be divided into two categories: specific and nonspecific [1-3]. Specific low back pain can be induced by apparent causes such as infection

Received Jun 9, 2017; Revised Jul 4, 2017; Accepted Jul 23, 2017

Corresponding author: Tetsuhiro Ishikawa

Department of Orthopaedic Surgery, Graduate School of Medicine, Chiba University, 1-8-1 Inohana, Chuo-ku, Chiba, 260-8670, Japan Tel: +81-43-226-2117, Fax: +81-43-226-2116, E-mail: ishikawa_tetsuhiro@yahoo.co.jp

*These authors contributed equally to this work. 
or spinal tumors [3]. However, $80 \%$ to $90 \%$ of low back pain cases are classified as nonspecific, without identifiable causes; nonspecific low back pain is often chronic and difficult to treat $[4,5]$. Intervertebral disc (IVD) pathology may be one significant contributor of low back pain, and early signs of IVD degeneration are proteoglycan loss, dehydration, and collagen degradation [3,6-8]. Magnetic resonance imaging (MRI) has been widely used to detect IVD degeneration because it is noninvasive and useful for evaluating soft tissue [9]. The Pfirrmann grading system, which is a semiquantitative classification of IVD degeneration using T2-weighted MRI, has been widely used to assess IVD degenerative changes, but some authors have reported that the Pfirrmann grading system cannot detect early IVD degeneration [10-12].

Proteoglycan is a hydrophilic macromolecule that leads to the hydration of the nucleus pulposus, accounting for the load-bearing property of the disc [11-17]. There are few reports that axial T2 mapping can provide a useful and noninvasive evaluation of the matrix status in IVDs and can be a useful indication of disc function [9]. Recent clinical studies have explored the use of T1 $\rho$ mapping to evaluate symptomatic IVD degeneration [11-17]. Recently, T1 $\rho$ evaluation has become the focus of attention; therefore, the purpose of this study was to investigate the correlation between $\mathrm{T} 1 \rho / \mathrm{T} 2$ mapping and degenerative grades and histological findings in a rabbit IVD injury model.

\section{Materials and Methods}

All protocols for animal procedures were approved by the Ethics Committees of Chiba university (approval no., 2-280) following the National Institutes of Health Guidelines for the Care and Use of Laboratory Animals (1996 version).

\section{Disc injury model}

IVD degeneration was induced via a well-established rabbit annular puncture model [18-20]. Under general anesthesia using ketamine hydrochloride $(10 \mathrm{mg} / \mathrm{kg}$, Ketalar; Daiichi Sankyo Co. Ltd., Tokyo, Japan), rabbits ( $\mathrm{n}=12$, New Zealand White, 8-week old) were placed in the lateral prone position. The posterolateral retroperitoneal approach was used to expose the anterolateral surface of three consecutive lumbar IVDs (L2/3 to L4/5). Annular punctures were made 10 times at L2/3, 5 times at L3/4, and 1 time at $\mathrm{L} 4 / 5$ using an 18-gauge needle $(\mathrm{n}=6)$ or a 21-gauge needle $(n=6)$. The tip of the needle was marked $5 \mathrm{~mm}$ from the tip, and the needle was inserted to a depth of $5 \mathrm{~mm}$ at the center of the disc level through the annulus fibrosus into the nucleus pulposus. A small amount of gelatinous nucleus pulposus tissue was observed at the core of the needle. The nonpunctured discs L1/2 and L5/6 served as normal control discs.

\section{Magnetic resonance imaging}

The rabbits were euthanized four $(n=6)$ and eight $(n=6)$ post-surgery using an overdose intraperitoneal injection of ketamine hydrochloride. Immediately after euthanasia, MRI was performed using a 1.5-Tesla scanner (Signa HDx; GE Healthcare, Milwaukee, WI, USA) with a transmit-receive eight-channel knee coil.

High-resolution T2-weighted morphological images were acquired in the sagittal orientation prior toT $1 \rho$ and T2 mapping to select a slice that passed through the center of the IVD. T1 $\rho$ measurement was performed at the selected slice by three-dimensional spoiled gradient-recalled echo sequencing using the following parameters: echo time/repetition time $(\mathrm{TE} / \mathrm{TR})=3 / 6 \mathrm{~ms}$; time of spin-lock $(\mathrm{TSL})=0,20,40$, and $60 \mathrm{~ms}$; spin-lock frequency $=500 \mathrm{~Hz}$, resolution $=0.36 \times 0.36 \times 3 \mathrm{~mm}^{3}$, and acquisition time $=10$ minutes. T2 measurement was performed at the selected slice by morphological imaging using two-dimensional multi-spin echo (MSE) sequence with the following parameters: TE/TR $=10-90$ ( 8 echoes $) / 1,500 \mathrm{~ms}$, in-plane resolution $=0.36 \times 0.36 \mathrm{~mm}^{2}$, slice thickness $=3 \mathrm{~mm}$, and acquisition time $=9$ minutes.

T1 $\rho$ - and T2-calculated maps were generated using MATLAB software (Mathworks, Natick, MA, USA) with a monoexponential curve fit; SI (image intensity) (TSL)=S0 $\left(\right.$ spin-density) ${ }^{*} \exp (-\mathrm{TSL} / \mathrm{T} 1 \rho)$ and $\mathrm{SI}=\mathrm{S} 0^{*} \exp (-\mathrm{TE} / \mathrm{T} 2)$, respectively. To minimize error from stimulated echoes in the T2 measurement, the first echo was excluded from the MSE sequence. Using MATLAB, color-coded T1 $\rho$ - and T2-calculated maps of the IVDs were created. An elliptical region of interest was manually positioned in the center of the IVD as follows: to exclude the noisy area, the upper and lower one-pixel thickness of each disc was removed; then, the discs were divided into three equal areas. The middle-third of the nucleus pulposus was designated by two authors (T.I. and M.M.). To standardize the proce- 
dure, all measurements were performed twice by both investigators and averaged. Intra- and interobserver agreements were assessed using percentages of agreement and kappa statistics. Mean T1 $\rho$ and T2 values were computed within the region.

\section{Tissue processing, grading, and image analysis}

Immediately after MRI, the lumbar discs were harvested and the slices were fixed in $4 \%$ paraformaldehyde ( $\mathrm{pH} 7.4$ ) for 48 hours. The discs were subsequently decalcified in $0.1 \mathrm{~mol} / \mathrm{L}$ EDTA (ethylenediaminetetraacetic acid, $\mathrm{pH}$ 7.2) over a 4- to 10-week period, depending on the calcification of the osseous matrix of the vertebral bone. The decalcified complete sagittal disc slices were then embedded into paraffin. The slices were then macroscopically evaluated, and two authors (T.I. and M.M.) macroscopically assessed the degree of disc degeneration using the Thompson grading system [21]. The IVDs were classified as follows: grade 1: normal; grade 2: mild disc degeneration; grade 3: moderate disc degeneration; grade 4: severe; and grade 5: severe with osteophytes of $>2 \mathrm{~mm}$ [21]. Both observers independently graded the degenerative discs twice with approximately one month between grading. Results from the first classifi- cation were used to evaluate interobserver agreement, and the level of agreement was assessed by kappa values. From the resulting blocks, paraffin sections were cut $(2-4 \mu \mathrm{m}$ thick) and placed on silanized glass slides. All specimens were treated for hematoxylin and eosin, safranin-O, and toluidine blue staining.

\section{Statistics}

Disc degeneration grades and histologic findings were compared using T1 $\rho$ and T2 values of each IVD by nonrepeated measures analysis of variance and the StudentNewman-Keuls method. Correlation analysis of MRI and histology was performed using Spearman's correlation test. Statistical significance was set as $p<0.05$. Statistical software (JMP, ver. 9.0; SAS Institute Inc., Cary, NC, USA) and Microsoft Excel (Microsoft, Redmond, WA, USA) were used for analysis.

\section{Results}

Intra- and interobserver agreements with Thompson grades are summarized in Table1. The level of agreement was assessed by kappa values following criteria reported
(A)

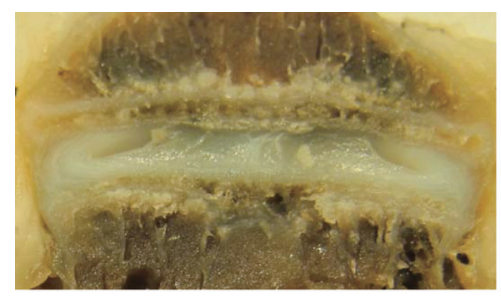

Normal

Fig. 1. The intervertebral discs of each group. Disc height decreased using the larger needle (black arrow) as the number of punctures increased (white arrow). (A) Normal, (B) $21 \mathrm{G}$ needle (1, 5, and 10 punctures), and (C) $18 \mathrm{G}$ needle (1, 5, and 10 punctures). G, gauge.
(B)

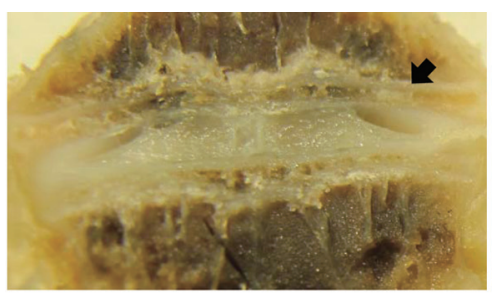

21G-1 puncture

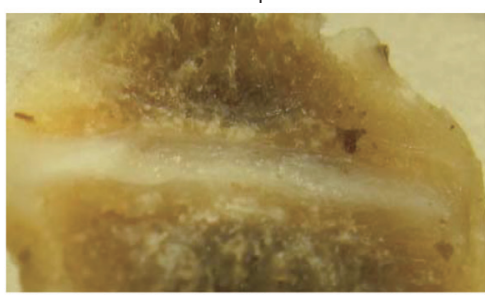

21G-5 punctures

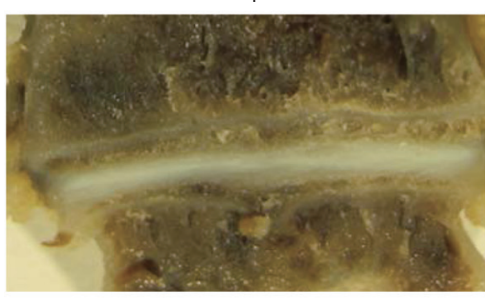

21G-10 punctures
(C)

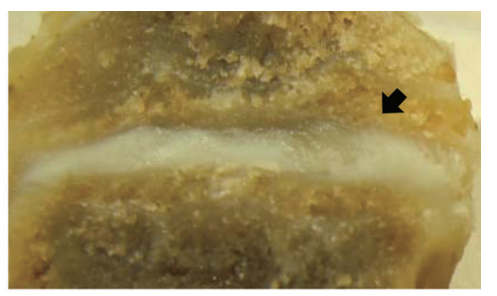

18G-1 puncture

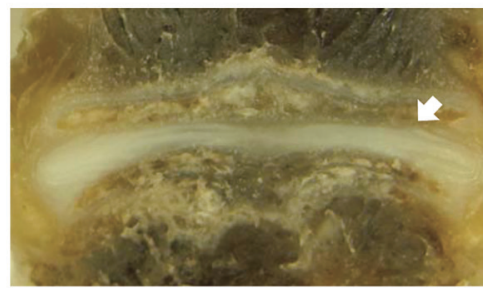

18G-5 punctures

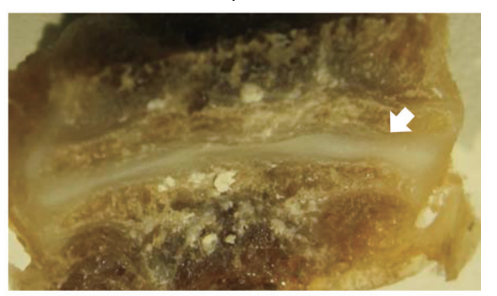

18G-10 punctures 
by Landis and Koch [22]: a negative kappa value was interpreted as poor agreement, $0.00-0.20$ as slight agreement, $0.21-0.40$ as fair agreement, $0.41-0.60$ as moderate agreement, $0.61-0.80$ as substantial agreement, and $>0.81$ as almost perfect agreement.

The IVDs were categorized as Thompson grade $1(\mathrm{n}=24)$, grade $2(n=14)$, grade $3(n=16)$, grade $4(n=6)$, and grade $5(\mathrm{n}=0)$. Compared to the normal IVDs, the injured IVDs showed a decrease in disc height and the degeneration of annulus fibrosus; this was macroscopically observed. The consequent disc degeneration became more severe as the number of punctures increased and when the larger needle was used (Fig. 1).

In the histological study, compared to the normal IVDs, the injured IVDs showed a decrease in disc height, consolidation of fibrous tissue in the nucleus pulposus, and cartilage disorganization of the annulus fibrosus (Fig. 2). (A, D, G, J: normal disc; B, E, H, K: disc of the 4-week group punctured by the 21-gauge needle; C, F, I, L: disc of the 8-week group punctured by the 18-gauge needle; A, B, C, J, K, L: hematoxylin and eosin staining; D, E, F: safraninO staining; G, H, I: toluidine blue staining). Compared to
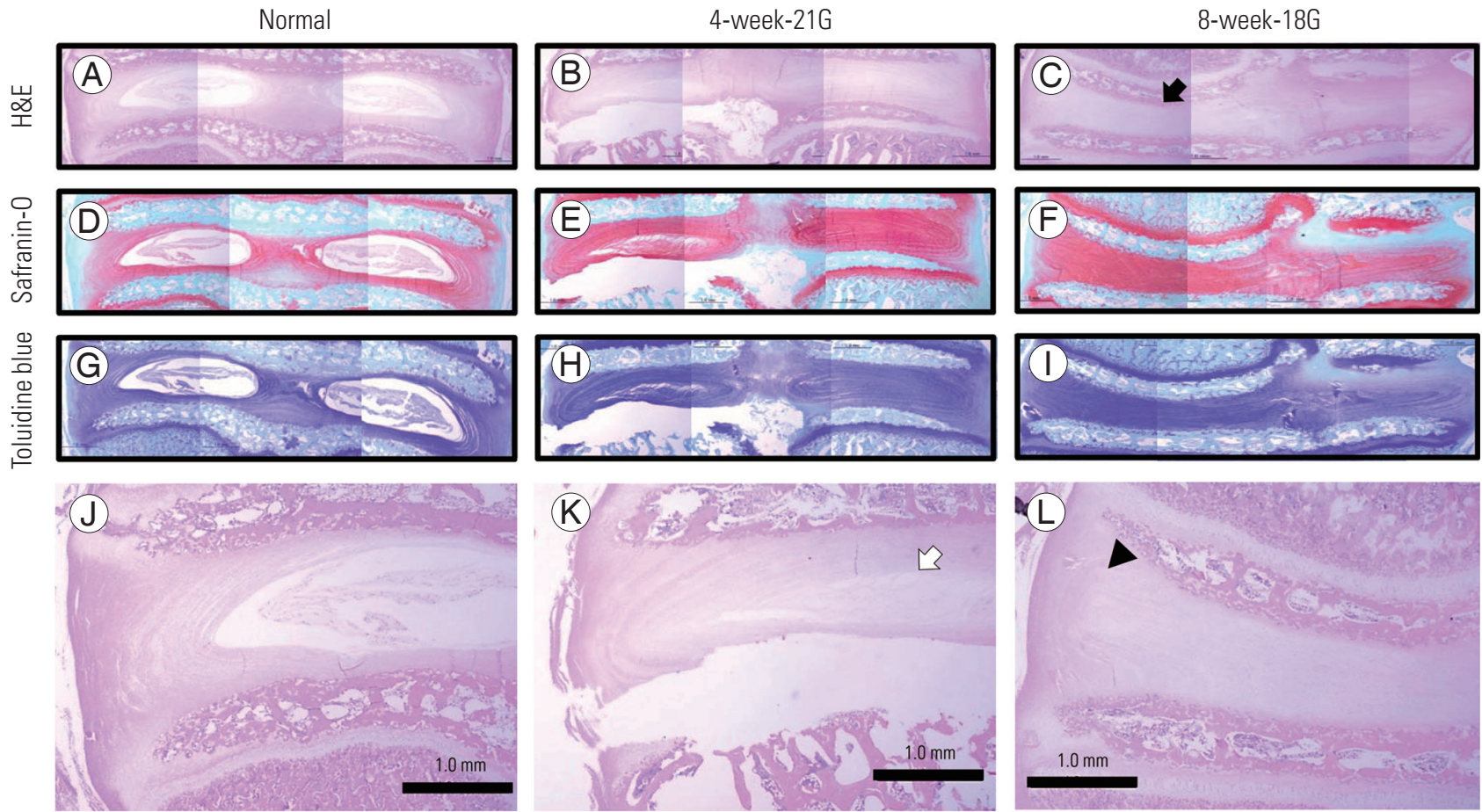

Fig. 2. (A-L) Representative histology of the intervertebral disc. G, gauge.
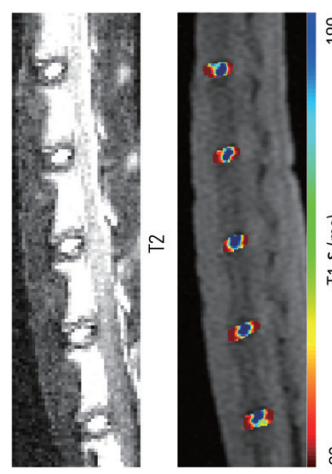

(A) Normal
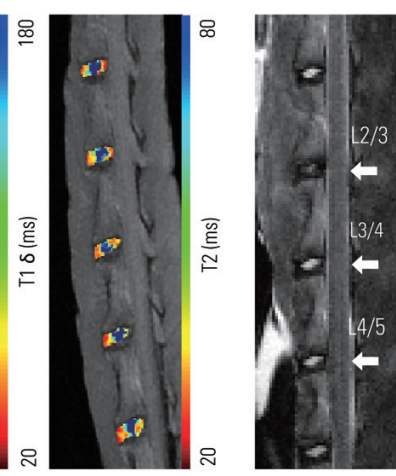

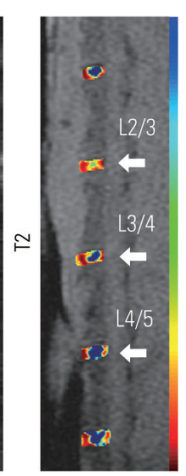

(B) 4-week-21G
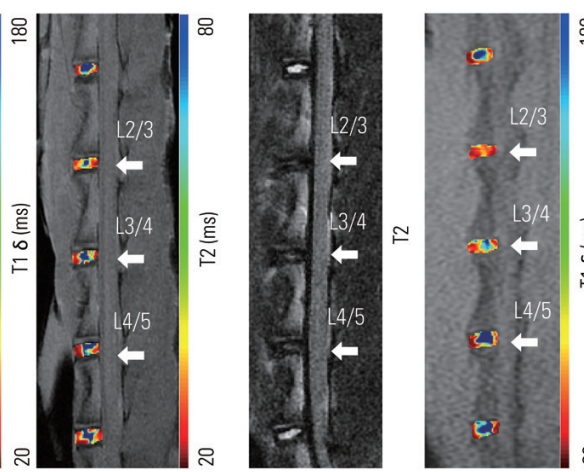

C 8 -week-18G

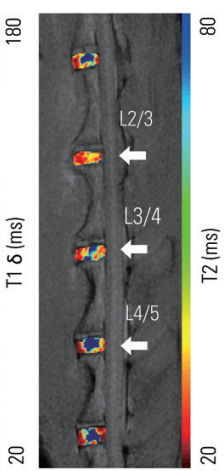

焉

Fig. 3. (A-C) Representative color maps with different grades of injury. Punctured intervertebral discs are indicated as arrows. Blue represents areas of short T1 $\rho$ and $\mathrm{T} 2$ values, and red represents areas of long T1 $\rho$ and T2 values. G, gauge. 
Table 1. Intra- and interobserver agreement of the Thompson grades

\begin{tabular}{lcc} 
Variable & Agreement (\%) & $\kappa$ \\
Intraobserver & 77.8 & 0.69 \\
Interobserver & & \\
T.I. vs. T.I. & 77.8 & 0.66 \\
M.M. vs. M.M. & 83.3 & 0.77 \\
\hline
\end{tabular}

the normal controls, the injured IVDs showed decreased disc height (black arrow), consolidation of fibrous tissue in the nucleus pulposus (white arrow), and cartilage disorganization within the annulus fibrosus (black arrowhead). As in the macroscopic study, these changes became more

Table 2. Mean T1 $\rho$ and T2 values in each Thompson grade

\begin{tabular}{lccc} 
Thompson grade & T1 $\rho$ value $(\mathrm{ms})$ & T2 value $(\mathrm{ms})$ & No. \\
1 & $179.3 \pm 33.7$ & $65.5 \pm 9.5$ & 24 \\
\hline 2 & $144.8 \pm 48.7$ & $59.0 \pm 9.3$ & 14 \\
\hline 3 & $98.9 \pm 7.4$ & $45.2 \pm 9.5$ & 16 \\
4 & $76.1 \pm 10.5$ & $38.3 \pm 4.4$ & 6 \\
\hline 5 & - & - & 0 \\
\hline
\end{tabular}

severe as the number of punctures increased and when the larger needle was used (Fig. 2).

Representative T1 $\rho$ and T2 color maps of the different grades of injury overlaid on magnetic resonance (MR) images with T2-weighted images are shown in Fig. 3. Punctured discs are indicated by arrows. The mean T1 $\rho$ values in the IVDs are as follows: grade 1: $179.3 \pm 33.7 \mathrm{~ms}$, grade 2: $144.8 \pm 48.7 \mathrm{~ms}$, grade $3: 98.9 \pm 7.4 \mathrm{~ms}$, and grade 4 : $76.1 \pm 10.5 \mathrm{~ms}$ (Table 2). The mean T2 values in the IVDs are as follows: grade 1: $65.5 \pm 9.5 \mathrm{~ms}$, grade $2: 59.0 \pm 9.3 \mathrm{~ms}$, grade 3: $45.2 \pm 9.5 \mathrm{~ms}$, and grade $4: 38.3 \pm 4.4 \mathrm{~ms}$ (Table 2). $\mathrm{T} 1 \rho$ and $\mathrm{T} 2$ values decreased as the grade of degeneration increased.

Figs. 4 and 5 display the median T1 $\rho$ and T2 values of the IVDs, respectively. There were significant differences between grade 1 and grade 2 IVDs $(p<0.05)$, grade 1 and grade 3 IVDs ( $p<0.01)$, grade1 and grade 4 IVDs $(p<0.01)$, grade 2 and grade 3 IVDs $(p<0.05)$, and grade 2 and grade 4 IVDs $(p<0.05)$ in terms of T1 $\rho$ values (Fig. 4$)$ and between grade 1 and grade 3 IVDs $(p<0.01)$, grade 1 and grade 4 IVDs $(p<0.01)$, grade 2 and grade 3 IVDs $(p<0.01)$, and grade 2 and grade 4 IVDs $(p<0.01)$ in terms of T2

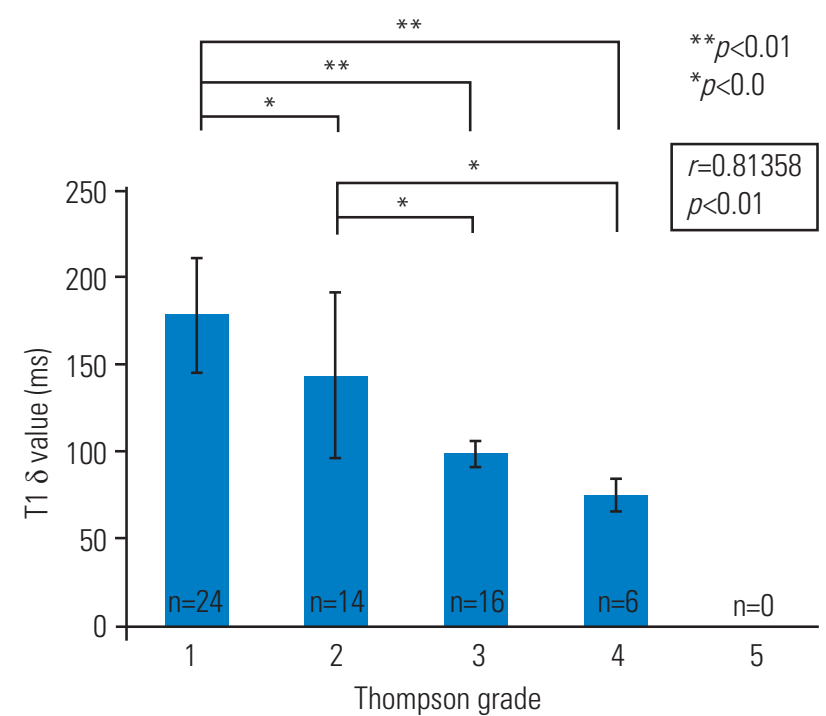

Fig. 4. Mean T1 $\rho$ values ( \pm standard deviation) for each Thompson grade. Regression analysis showed a negative linear relationship of the Thompson grade with $T 1 \rho$ values $(p<0.05)$.

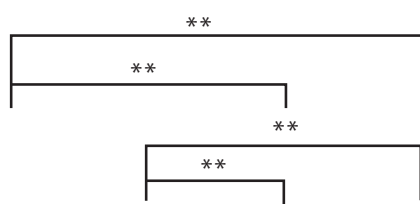

${ }^{* *} p<0.01$

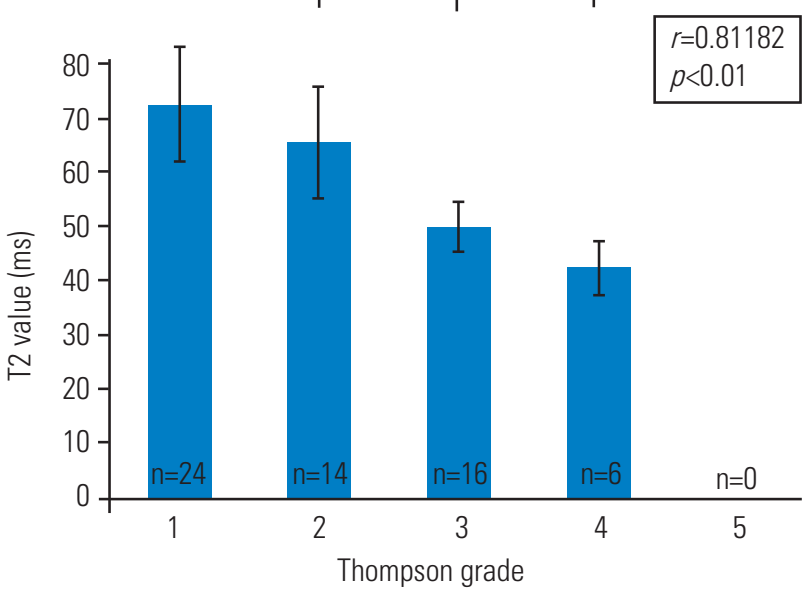

Fig. 5. Mean T2 values ( \pm standard deviation) for each Thompson grade. Regression analysis showed a negative linear relationship of the Thompson grade with $\mathrm{T} 2$ values $(p<0.05)$.

values (Fig. 5). There was a significant difference between grade 1 and grade 2 IVDs only in terms of T1 $\rho$ values (Fig. $4)$. Correlation analysis showed a positive linear relationship of Thompson grades with T1 $\rho$ values $(p<0.05)$ and $\mathrm{T} 2$ values $(p<0.05)$ (Figs. 4,5$)$. T1 $\rho$ and T2 values in each IVD and the Thompson grade of each IVD are indicated in Table 3. 
Table 3. The Thompson grade of each intervertebral disc

\begin{tabular}{|c|c|c|c|c|c|c|}
\hline Size of the needle & No. of punctures & Grade 1 & Grade 2 & Grade 3 & Grade 4 & Grade 5 \\
\hline \multirow[t]{3}{*}{18 gauge } & 10 & 0 & 0 & 2 & 4 & 0 \\
\hline & 5 & 0 & 2 & 2 & 2 & 0 \\
\hline & 1 & 2 & 2 & 2 & 0 & 0 \\
\hline \multirow[t]{3}{*}{21 gauge } & 10 & 0 & 0 & 6 & 0 & 0 \\
\hline & 5 & 0 & 2 & 4 & 0 & 0 \\
\hline & 1 & 4 & 2 & 0 & 0 & 0 \\
\hline Control & - & 18 & 6 & 0 & 0 & 0 \\
\hline
\end{tabular}

\section{Discussion}

The present study investigated the effectiveness of quantitative T1 $\rho / T 2$ MRI of the IVD and evaluated the associations between MRI findings (T1 $\rho$ and T2), imaging parameters (Thompson grade), and histological changes. Three primary findings were obtained. First, though not significant, disc degeneration became more severe as the number of punctures increased and when the larger needle was used. Second, both T1 $\rho$ and T2 quantitative MRI could detect these small differences, and these degenerative changes were also observed in the histological study. Third, T1 $\rho$ mapping seems to have an advantage over T2 mapping for detecting the early degenerative changes of IVDs.

A rabbit model of disc degeneration by an annular needle puncture was established by Masuda et al. [18] to induce slow, mild, and reproducible disc degeneration $[19,20]$. Extrusion of the nucleus pulposus made by a 5 -mm deep needle puncture induces disc degeneration in this model; this was subsequently validated histologically, radiographically, and by MRI [19]. In the current study, during both macroscopic and histologic examinations, compared to the normal IVDs, we observed a decrease in disc height and degeneration of the annulus fibrosus in the injured IVDs. IVD degeneration became more severe as the number of punctures increased and when the larger needle was used. These results suggest that we are able to control the degree of IVD degeneration in this model.

In the present study, intra- and interobserver errors were small as indicated by kappa values for Thompson grades. This result indicates that Thompson grades are appropriate measures for evaluating IVD degenerative changes in this model.

Although T1 $\rho$ and T2 values were significantly different between grade 1 or grade 2 IVDs and grade 3 and grade 4 IVDs, there was a significant difference between grade 1 and grade 2 IVDs only in terms of T1 $\rho$ values (Fig. 3). T1 $\rho$ values in grade 2 IVDs were significantly lower than those in grade 1 IVDs. These data suggest that T1 $\rho$ mapping is more sensitive to early degenerative changes than T2 mapping. Nguyen et al. [14] demonstrated that T1 $\rho$-weighted MRI is directly correlated with isometric swelling pressure of cadaveric discs and suggested that it is a valuable quantitative biomarker of the mechanical function of the nucleus pulposus. Zobel et al. [11] reported that T1 $\rho$ values correlate with the Pfirrmann degenerative grade in young adults; however, the Pfirrmann grading system could not detect early degenerative changes. The Pfirrmann grading system is a semiquantitative assessment of morphologic IVD degeneration using T2-weighted MRI $[10,17]$. T1 $\rho$ mapping might be useful for assessing early degenerative changes. Blumenkrantz et al. [12] observed a correlation between T1 $\rho$ mapping and clinical findings quantified by the 36-Item Short Form Health Survey and Oswestry Disability Index in 16 patients. T1 $\rho$ values also correlated with patient-reported physical activity and disability [12]. Zuo et al. [16] reported that magnetic resonance spectroscopy (MRS)-quantified water, MRS-quantified proteoglycan (PG), water/PG ratio, and the Pfirrmann grade were significantly associated with clinical self-assessment and that the T1 $\rho$ value was significantly associated with MRSquantified PG content. Another reason why T1 $\rho$ mapping may be more sensitive than T2 mapping is that T1 $\rho$ mapping has a higher dynamic range for detecting early pathology. This higher dynamic range can be exploited to more accurately measure even small macromolecular changes $[15,16]$. Therefore, there is a possibility that T1 $\rho$ mapping is sensitive to early degenerative changes and correlates with clinical symptoms in patients with IVD 
degeneration.

Our results showed a significant positive linear relationship of Thompson grades with T1 $\rho$ and T2 values. Previous studies conducted using cartilage have shown that T2 mapping is highly related to collagen integrity while it is not very sensitive to changes in macromolecules such as PG $[23,24]$. Axial T2 mapping can provide a useful noninvasive evaluation of the matrix status in IVDs and be a useful indicator of disc function [25]. Thus, assessing IVD degeneration using $\mathrm{T} 1 \rho$ and $\mathrm{T} 2$ mapping may increase our current understanding of degenerative processes. IVD assessment using T1 $\rho$ mapping will help evaluate new therapies for treating IVD degeneration, such as tissue engineering strategies, recombinant growth factor injection, gene therapies, and stem cell therapies [26-30].

There are some limitations to this study. First, we only assessed the status of IVDs at 4 and 8 weeks post injury. These early time points after injury are important for evaluating early degenerative changes. Second, different sequences were used for the two MR parameter measurements: spin-preparation spoiled gradient-recalled echo in T1 $\rho$ mapping and multi-echo spin echo in T2 mapping. Depending on the MR acquisition method, MR parameters and the sensitivity to degeneration can be changed.

\section{Conclusions}

The evaluation of IVD degeneration using T1 $\rho$ and T2 mapping may be a future clinical tool for identifying IVD degeneration in patients. T1 $\rho$ mapping might be more sensitive than T2 mapping for evaluating early degenerative changes in lumbar IVDs. Though the relevance of early degenerative changes in humans is controversial, T1 $\rho$ mapping as a new emerging MRI technique might be more relevant for basic research such as biological disc treatment and cell therapy approaches. Consequently, specimens obtained using this method may be easier to analyze than human tissues.

\section{Conflict of Interest}

No potential conflict of interest relevant to this article was reported.

\section{ORCID}

Tetsuhiro Ishikawa: https://orcid.org/0000-0001-7413-6566
Atsuya Watanabe: https://orcid.org/0000-0003-2804-4876

Hiroto Kamoda: https://orcid.org/0000-0001-8982-2278

Masayuki Miyagi: https://orcid.org/0000-0002-3082-9739

Gen Inoue: https://orcid.org/0000-0001-6500-9004

Kazuhisa Takahashi: https://orcid.org/0000-0002-1707-6656

Seiji Ohtori: https://orcid.org/0000-0001-5738-6184

\section{References}

1. Andersson GB. Epidemiological features of chronic low-back pain. Lancet 1999;354:581-5.

2. Wipf JE, Deyo RA. Low back pain. Med Clin North Am 1995;79:231-46.

3. Takahashi K, Aoki Y, Ohtori S. Resolving discogenic pain. Eur Spine J 2008;17 Suppl 4:428-31.

4. Nachemson AL. The lumbar spine an orthopaedic challenge. Spine 1976;1:59-71.

5. Mooney V. Presidential address: International Society for the Study of the Lumbar Spine; Dallas, 1986; where is the pain coming from? Spine (Phila $\mathrm{Pa}$ 1976) 1987;12:754-9.

6. Deyo RA, Weinstein JN. Low back pain. N Engl J Med 2001;344:363-70.

7. Raj PP. Intervertebral disc: anatomy-physiologypathophysiology-treatment. Pain Pract 2008;8:18-44.

8. Adams MA, Roughley PJ. What is intervertebral disc degeneration, and what causes it? Spine (Phila $\mathrm{Pa}$ 1976) 2006;31:2151-61.

9. Watanabe A, Benneker LM, Boesch C, Watanabe T, Obata T, Anderson SE. Classification of intervertebral disk degeneration with axial T2 mapping. AJR Am J Roentgenol 2007;189:936-42.

10. Pfirrmann CW, Metzdorf A, Zanetti M, Hodler J, Boos N. Magnetic resonance classification of lumbar intervertebral disc degeneration. Spine (Phila $\mathrm{Pa}$ 1976) 2001;26:1873-8.

11. Zobel BB, Vadala G, Del Vescovo R, et al. T1 $\rho$ magnetic resonance imaging quantification of early lumbar intervertebral disc degeneration in healthy young adults. Spine (Phila Pa 1976) 2012;37:1224-30.

12. Blumenkrantz G, Zuo J, Li X, Kornak J, Link TM, Majumdar S. In vivo 3.0-tesla magnetic resonance T1rho and T2 relaxation mapping in subjects with intervertebral disc degeneration and clinical symptoms. Magn Reson Med 2010;63:1193-200.

13. Johannessen W, Auerbach JD, Wheaton AJ, et al. Assessment of human disc degeneration and proteo- 
glycan content using T1rho-weighted magnetic resonance imaging. Spine (Phila Pa 1976) 2006;31:12537.

14. Nguyen AM, Johannessen W, Yoder JH, et al. Noninvasive quantification of human nucleus pulposus pressure with use of T1rho-weighted magnetic resonance imaging. J Bone Joint Surg Am 2008;90:796802.

15. Blumenkrantz G, Li X, Han ET, et al. A feasibility study of in vivo T1rho imaging of the intervertebral disc. Magn Reson Imaging 2006;24:1001-7.

16. Zuo J, Joseph GB, Li X, et al. In vivo intervertebral disc characterization using magnetic resonance spectroscopy and T1 $\rho$ imaging: association with discography and Oswestry Disability Index and Short Form36 Health Survey. Spine (Phila Pa 1976) 2012;37:21421.

17. Roughley PJ, Alini M, Antoniou J. The role of proteoglycans in aging, degeneration and repair of the intervertebral disc. Biochem Soc Trans 2002;30(Pt 6):869-74.

18. Masuda K, Aota Y, Muehleman C, et al. A novel rabbit model of mild, reproducible disc degeneration by an annulus needle puncture: correlation between the degree of disc injury and radiological and histological appearances of disc degeneration. Spine (Phila Pa 1976) 2005;30:5-14.

19. Aoki Y, Akeda K, An H, et al. Nerve fiber ingrowth into scar tissue formed following nucleus pulposus extrusion in the rabbit anular-puncture disc degeneration model: effects of depth of puncture. Spine (Phila Pa 1976) 2006;31:E774-80.

20. Obata S, Akeda K, Imanishi T, et al. Effect of autologous platelet-rich plasma-releasate on intervertebral disc degeneration in the rabbit anular puncture model: a preclinical study. Arthritis Res Ther 2012;14:R241.
21. Thompson JP, Pearce RH, Schechter MT, Adams ME, Tsang IK, Bishop PB. Preliminary evaluation of a scheme for grading the gross morphology of the human intervertebral disc. Spine (Phila Pa 1976) 1990;15:411-5.

22. Landis JR, Koch GG. The measurement of observer agreement for categorical data. Biometrics 1977;33:159-74.

23. Kettler A, Wilke HJ. Review of existing grading systems for cervical or lumbar disc and facet joint degeneration. Eur Spine J 2006;15:705-18.

24. Xia Y, Farquhar T, Burton-Wurster N, Lust G. Origin of cartilage laminae in MRI. J Magn Reson Imaging 1997;7:887-94.

25. Regatte RR, Akella SV, Borthakur A, Kneeland JB, Reddy R. Proteoglycan depletion-induced changes in transverse relaxation maps of cartilage: comparison of T2 and T1rho. Acad Radiol 2002;9:1388-94.

26. Masuda K. Biological repair of the degenerated intervertebral disc by the injection of growth factors. Eur Spine J 2008;17 Suppl 4:441-51.

27. Vadala G, Sowa G, Hubert M, Gilbertson LG, Denaro V, Kang JD. Mesenchymal stem cells injection in degenerated intervertebral disc: cell leakage may induce osteophyte formation. J Tissue Eng Regen Med 2012;6:348-55.

28. Vadala G, Mozetic P, Rainer A, et al. Bioactive electrospun scaffold for annulus fibrosus repair and regeneration. Eur Spine J 2012;21 Suppl 1:S20-6.

29. Moon CH, Jacobs L, Kim JH, et al. Part 2: quantitative proton $\mathrm{T} 2$ and sodium magnetic resonance imaging to assess intervertebral disc degeneration in a rabbit model. Spine (Phila Pa 1976) 2012;37:E1113-9.

30. Saar G, Zhang B, Ling W, Regatte RR, Navon G, Jerschow A. Assessment of glycosaminoglycan concentration changes in the intervertebral disc via chemical exchange saturation transfer. NMR Biomed 2012;25:255-61. 